\title{
Internet : un nouveau terrain d'enquête
}

Quelques pistes à explorer d'après une expérience

\section{Marie-Jo Derive}

\section{(2) OpenEdition}

\section{Journals}

Édition électronique

URL : https://journals.openedition.org/clo/114

DOI : $10.4000 /$ clo. 114

ISSN : 2266-1816

Éditeur

INALCO

\section{Édition imprimée}

Date de publication : 1 janvier 2008

Pagination : 117-125

ISBN : 978-2-85831-181-1

ISSN : 0396-891X

Référence électronique

Marie-Jo Derive, «Internet : un nouveau terrain d'enquête », Cahiers de littérature orale [En ligne], 63-64 | 2008, mis en ligne le 28 décembre 2011, consulté le 07 juillet 2021. URL : http://

journals.openedition.org/clo/114; DOI : https://doi.org/10.4000/clo.114

Ce document a été généré automatiquement le 7 juillet 2021.

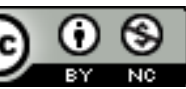

Cahiers de littérature orale est mis à disposition selon les termes de la Licence Creative Commons Attribution - Pas d'Utilisation Commerciale 4.0 International. 


\title{
Internet : un nouveau terrain d'enquête
}

\author{
Quelques pistes à explorer d'après une expérience
}

\author{
Marie-Jo Derive
}

1 Sollicités par une de nos collègues ${ }^{1}$ pour participer à un colloque sur les insultes rituelles et leurs fonctions culturelles, nous pensions, Jean Derive et moi, présenter une communication à partir de celles que nous avions pu relever lors de notre séjour en Côte d'Ivoire de 1975 à 1979. Grâce à une discussion avec Nguettia Kouadio Kobenan, doctorant ivoirien à l'université de Savoie à cette époque, nous découvrîmes alors un terrain d'enquête complémentaire et très original pour enrichir notre corpus. Il s'agissait d'un site Internet appelé «gate-gate » sur lequel des membres dispersés de la diaspora ivoirienne se livraient au jeu du même nom.

\section{De quoi est-il question?}

2 Le gate-gate est un jeu rituel qui se pratique couramment in praesentia, entre autres jeux verbaux qui constituent l'un des fonds de la littérature orale ludique ivoirienne. Car c'est bien en l'occurrence de littérature orale qu'il s'agit dans la mesure où il existe déjà un répertoire patrimonial et où les créations qui viennent l'enrichir obéissent à des règles canoniques et poétiques précises².

3 La dénomination de ce jeu a son origine dans une expression en nouchi ${ }^{3}$, variété $d u$ français populaire d'Abidjan parlé par les jeunes, dans laquelle "insulter» se dit " gâter ${ }^{4}$ le nom ». Il s'agit d'un duel où chaque partenaire cherche à " gâter » l'autre, à l'injurier dans un système de réparties $d u$ tac au tac suggéré par la répétition de «gate» de part et d'autre du tiret dans ce nom composé. Ce jeu se pratique surtout entre garçons et filles de quinze à vingt ans, qui se connaissent bien, devant trois ou quatre spectateurs; les injures portent essentiellement sur les parties physiques de la personne avec interdiction toutefois d'évoquer son handicap réel au cas où elle en aurait un. Le gagnant est celui (ou celle) qui épuise le répertoire ou les possibilités d'inventivité de l'autre. On peut aussi s'arrêter quand on en a envie. 
4 Le gate-gate, très prisé par les jeunes à Abidjan, est apparu sur le Net en 1999, comme le précisait le site http://www.nouchi.com en 2003. Pour analyser «les processus de création et valeur d'emploi des insultes en français populaire de Côte d'Ivoire », ce qui était notre objectif pour ce colloque, nous avons imprimé les échanges recueillis sur le net en juillet, août, septembre 2002, soit huit cent quarante-huit relevés sur cinquante pages imprimées ${ }^{5}$.

5 Ce type de recherche complètement nouveau pour nous et découvert un peu par hasard pose au chercheur un certain nombre de questions, notamment: quelles sont les nouvelles conditions d'une enquête de ce genre sur Internet et qu'est-ce que l'analyse des résultats permet d'en faire? C'est ce que nous nous proposons très brièvement de présenter.

\section{Les nouvelles conditions du jeu}

6 La première remarque que l'on peut faire à propos des conditions d'énonciation de ce jeu recueilli sur Internet est que, contrairement aux habituelles études de terrain telles que celle par exemple décrite par J. Derive dans ce même numéro, est que tous les acteurs, y compris l'observateur lui-même, sont cachés ou du moins ne sont pas présents physiquement. Ce fut en tout cas le parti que nous avons choisi pour l'observation que nous voulions faire. Nous pouvons déjà souligner l'un des avantages que présente ce type de recherche par rapport à l'observation habituelle : la garantie d'authenticité maximale dans le déroulement du jeu sans qu'il soit perturbé par la présence du chercheur au moment de son énonciation; les données sont ainsi recueillies dans leur état brut. Ensuite, comme tout matériau recueilli sur le terrain, il pourra être expliqué, commenté dans un deuxième temps avec un informateur (en l'occurrence pour nous Nguettia Kouadio qui connaissait à la fois le nouchi et le jeu).

7 Cette présence virtuelle des acteurs eux-mêmes modifie forcément le jeu lui-même puisque d'une part les spectateurs, s'il y en a, sont cachés, mais surtout ses règles sont également changées: la pratique du jeu n'est plus seulement duelle, mais plurielle avec le net tous ceux qui connaissent et le jeu et l'adresse du site participent; nous verrons plus loin les autres modifications que cela entraîne. Quoi qu'il en soit, l'essentiel du jeu demeure : l'échange d'insultes; celles-ci continuent à porter sur les traits physiques même si les acteurs ne se voient pas, comme en témoigne l'échange suivant :

John bri ${ }^{6}$ : toi gaou là tu te prends $\mathrm{s}^{7}$ pour qui avec ton visage on dirait gorille qui se masturbe avec matlatom.

8/30/2002 $6: 49: 58 \mathrm{AM}$

Crazy-d : ton crane qui brille on dirait tu as mis matlatom de tantie fatou dessus la... ta nuque on dirait climatiseur samsungla... ta pine on dirait cocotier de grand bassam petit joueur...

8/31/2002 $1: 53: 27$ PM

8 L'autre remarque concerne le jeu lui-même. Grâce à la possibilité offerte par Internet de transmettre dans l'immédiat des informations, la réactivité particulière qui caractérise ce type de communication ludique peut être conservée avec un léger décalage toutefois dans le temps comme le montrent les heures et jours des messages envoyés. Ce décalage modifie tout de même assez sensiblement un trait essentiel de ce jeu. Il permet aux partenaires de prendre éventuellement un temps de réflexion avant de répondre, ce qui déplace une partie de son intérêt fondé sur la présence d'esprit et 
l'art de la répartie du tac au tac. Cela dit, une partie de l'acte perlocutoire perdure : l'insulte déclenche une certaine agressivité qui est maintenue dans les propos sur le web, de même que le jeu l'implique en face à face.

Comme nous l'avons souligné plus haut, l'Internet ouvre ce jeu à tous les Ivoiriens dispersés dans le monde, qui sont connectés et qui le connaissent, ce qui suppose ainsi qu'on le voit dans certains commentaires, un rappel à l'ordre des règles du jeu :

Opray : les insultes ne sont pas jolies... Mais frerot ce site n'est pas là pour ça c juste pour se gater et je crois sincèrement ke je fais honneur aux règles dictées... tu devrais bien relire les pages et sans nul doute tu te rendras cpte de l'aberrance de ce ke tu dis.»

De même lorsque les échanges dévient, un des internautes ivoiriens rappelle :

L'inspectah : Votre gate-gate s'embourgeoise on dirait garden-party.

$8 / 13 / 2002$

Ou encore :

Opray : « Pauvre mauviette: Ce n'est qu'un jeu ici alors n'aie pas peur sinon sauvetoi et ne reviens plus. Enfoiré des égouts, nous nous chargerons de te trouver un nom, ok ».

$8 / 13 / 2002$

De nombreux autres exemples témoignent de cette nécessité de maintenir les règles, ainsi :

Spanish : «eh djah on a dit fo laissé affaire de vieille mère là fo arrêté de l'insulter elle est déjà préoccupée de ta présence sur le gate.»

$8 / 13 / 2002$

10 Le risque que ce gate-gate dégénère en banal « chat » sur Internet, où sont échangés des avis sur la politique ivoirienne, sur la nourriture dans d'autres pays que la Côte d'Ivoire, sur les femmes en général, des leçons de morale comme en témoignent divers échanges, est visiblement le souci des vrais joueurs qui, à maintes reprises, rappellent l'objectif de ce site :

Prouproulambert: merci pour cette intervention le cousin, nous sommes pas là pour parler du traintrain quotidien mais pour INSULTER !! ! ! ! !

\section{Quelques éléments d'analyse}

11 L'étude rapide de ces données recueillies par le biais de ce nouveau moyen de communication que représente l'Internet nous permet de faire quelques remarques à propos d'une nouvelle fonction sociolinguistique que revêt ce jeu d'insulte.

Quelle que soit la langue, ou le pays concerné, l'insulte est une chose particulièrement grave lorsqu'elle est dite dans le cadre d'une vraie querelle avec l'intention de blesser. Mais précisément parce qu'elle est très grave, la pratique de l'insulte a également donné lieu à des formes rituelles qui acquièrent alors une fonction cathartique. Cela est vrai à l'échelle de la parenté, de l'alliance matrimoniale, mais aussi pour d'autres formes d'alliance, entre ethnies voisines ou entre groupes sociaux d'une même ethnie. Puisqu'il est admis par convention entre ces partenaires bien définis qu'il est en quelque sorte recommandé de s'insulter " pour rire » d'une certaine façon l'insulte, la vraie, n'est plus possible. Un tel système ne peut plus fonctionner hors des repères socioculturels bien définis comme dans le cas de communautés urbaines où précisément ils ne sont plus visibles. Le besoin s'est sans doute alors fait sentir, pour canaliser la force de la pression sociale engendrée par l'insulte, de remplacer «l'alliance cathartique » traditionnelle par d'autres formes ritualisées, au caractère 
ludique affirmé. C'est probablement ainsi qu'est né le gate-gate, pratique typiquement urbaine au sein des jeunes, qui ne suppose plus une relation sociale prédéterminée, mais est fondée sur le consentement des partenaires de la communication qui affirment ainsi qu'ils «jouent à s'insulter ». La règle imposée de la gratuité à cet égard est significative.

En pratiquant le gate-gate, les partenaires affirment d'abord qu'ils partagent un code culturel à travers ce genre particulier de la communication: celui de structures énonciatives, de thèmes, de processus de figuration de l'insulte et éventuellement d'un répertoire prédéterminé. De facteur de discorde qu'elle était, l'insulte devient donc, dans ce cadre rituel, un facteur de solidarité mutuelle. Ceci est vrai, lorsqu'il est pratiqué in vivo dans les faubourgs d'Abidjan, mais à plus forte raison sur le net: on se reconnaît entre Ivoiriens par son aptitude à manier le gate-gate et on manifeste sa complicité dans la pratique même du rite, d'où l'insistance, sur le net, des joueurs à rappeler les règles du jeu, qui maintiennent ainsi à la fois l'identité ludique et l'identité culturelle. Ce besoin de reconnaissance d'une solidarité culturelle devient d'autant plus fort qu'on se trouve dans une situation d'acculturation. C'est ce qui explique sans doute le succès de ce jeu sur Internet où écrivent un grand nombre d'internautes appartenant à la diaspora ivoirienne dispersée aux quatre coins du monde. Coupés encore plus de leur communauté d'origine que ne le sont les habitants de Côte d'Ivoire, ces internautes entre lesquels l'insulte ne peut qu'être gratuite, puisqu'ils ne se connaissent pas et ne se voient pas, éprouvent d'autant plus la nécessité de partager ces signes de reconnaissance identitaire qui fondent entre eux un sentiment de solidarité.

14 Le succès de ce jeu est dû également à un nouveau mode d'expression, cette nouvelle «écriture " apparue avec Internet, très proche de l'oral par le code utilisé: l'orthographe, très souvent phonétique, comme on l'a vu dans les exemples, les onomatopées, la syntaxe conservée de cette variété de français populaire parlée en Côte d'Ivoire, les termes d'adresse pour apostropher le ou les destinataires :

Nervious: même sur internet vous êtes tous vilain on dirait diarrhée de phacochère vos nez on dirait caleçon de conan

Dans cet échange, un internaute dont le pseudonyme est " Nervious » au moyen du net comme il le mentionne, insulte plusieurs autres internautes en utilisant l'un des modèles formulaires qui domine de loin dans notre corpus, au point qu'il peut véritablement apparaître comme le modèle canonique de l'énoncé insultant en français populaire de Côte d'Ivoire. Ce modèle est construit sur le principe suivant : l'énoncé thématique d'un attribut de l'allocutaire insulté (qui fonctionne comme la métonymie de la personne), ici "vos nez", suivi d'un prédicat sur cet attribut qui consiste à l'assimiler à une image dépréciative "caleçon de conan", l'équation entre le comparant et le comparé se faisant au moyen de l'expression on dirait : la formule est la suivante :

« ton/ta ou votre/vos + attribut », « on dirait » + image dépréciative.

Ce type d'énoncé représente en effet à peu près $70 \%$ du corpus des six cents expressions utilisées dans les échanges que nous avons imprimés. Est-ce une caractéristique des insultes échangées au cours du gate-gate en général ou une spécificité de ce type d'échanges sur Internet? Nous ne pourrons répondre à cette question qu'en comparant avec un autre corpus recueilli cette fois in situ avec des partenaires réels. Cela dit, il est possible que la fixation par écrit entraîne un figement et une certaine standardisation des formules véhiculées sur le net. 
17 En cela, l'observation d'un corpus de ce genre est intéressante également du point de vue linguistique dans la mesure où on assiste, en direct en quelque sorte, à l'émergence d'une forme écrite d'une variété de langue, ce nouchi, cette variété de français populaire des jeunes d'Abidjan qui, jusque-là n'était utilisée qu'à l'oral. La grande tolérance des internautes par rapport à une norme orthographique traditionnelle facilite sans doute ce passage de l'oral à l'écrit.

\section{En forme de conclusion}

La ritualisation des échanges observés selon une forme canonique, décrite plus précisément dans notre article sur les injures, permet de répondre de façon positive à la question de savoir si ce type de matériau recueilli sur Internet constitue ou non une nouvelle forme de littérature orale. On pourrait être tenté en effet de considérer qu'il ne peut plus s'agir de "littérature orale » en arguant que, dans le cas du gate-gate sur Internet, les énoncés échangés sont écrits par les intéressés. Au pied de la lettre, l'échange n'est donc plus oral stricto sensu. Mais nous pensons avoir montré, au cours de ce témoignage, que cette nouvelle forme d'écriture, ni par son orthographe, souvent phonétique, ni par sa syntaxe n'obéit aux règles de l'écrit. De tels énoncés conservent plutôt la rhétorique de l'oralité. Cette illustration est par conséquent une occasion de mettre en évidence que le concept d'oralité ne doit pas être entendu de façon étroite. Il s'agit d'un esprit autant que d'un mode de transmission. Comme nous l'avons vu, dans le cas du gate-gate sur Internet, l'utilisation du média modifie un peu les règles du jeu (il ne saurait notamment plus y avoir de vainqueur puisque le plus souvent la relation n'est plus duelle et que la possibilité de différer tant soit peu le temps de réponse atténue l'effet tac au tac et ne permet plus aussi facilement de «clouer le bec » de l'adversaire), mais en revanche elle renforce la fonction hypocoristique et solidaire.

19 W. J. Ong (1982) et P. Zumthor (1983) ont depuis longtemps distingué une oralité première non médiatisée, et une oralité seconde, relayée par les médias. Internet, comme le montre l'exemple du gate-gate, peut être un des champs intéressants de cette oralité seconde.

Il est d'autant plus intéressant, dans le cas particulier de ce type de média qui permet des échanges très universels, qu'il témoigne d'un des aspects dont peut se construire une identité dans un monde globalisé, en combinant plusieurs types de références culturelles ${ }^{9}$.

\section{BIBLIOGRAPHIE}

BНАВНА, Homi K. 2007, Les lieux de la culture. Une théorie postcoloniale de la culture, Paris, Payot.

MEYRAN, Régis, 2008, Le rap à la conquête du monde, Sciences Humaines, nº 196. 
DERIVE, Marie-Jo et Jean, 2004, Processus de création et valeur d'emploi des insultes en français populaire de Côte d'Ivoire, in Dominique Lagorgette et Pierre Larrivée (éds), Les insultes : approches sémantiques et pragmatiques, Paris, Larousse, pp. 13-34.

LAGORGETTE, Dominique et Pierre LARRIVÉE (éds), 2004, Les insultes : approches sémantiques et pragmatiques, Langue française, $\mathrm{n}^{\circ}$ 144, Actes du colloque du 15 mars 2003, Paris, Larousse.

ONG, Walter J. 1982, Orality and Literacy. The Technologizing of the World, Londres, New York, Methuen.

ZuMTHOR, Paul, 1983, Introduction à la poésie orale, Paris, Seuil.

\section{NOTES}

1. Il s'agit de D. Lagorgette qui organisa le 15 mars 2003 un colloque international à l'université de Savoie dont les actes furent publiés (2004).

2. Voir à ce propos Marie-Jo et Jean Derive (2004).

3. Ce terme a déjà été signalé par Y. Simard (1994). L'origine de ce terme pourrait être du verlan et signifierait « chez nous », mais cette hypothèse n'a pas été vérifiée.

4. " Gâter » a ici le sens d' " abîmer » bien sûr.

5. À titre indicatif, ce jeu sur le net comportait plus de cent quarante-cinq pages à l'époque. Au moment de la rédaction de cet article, apparemment la page de ce jeu n'est plus accessible : le jeu a-t-il disparu?

6. Nous avons gardé le nom des pseudonymes tels qu'ils apparaissent sur le site.

7. Nous avons pris le parti de laisser l'orthographe, plus souvent proche de la phonétique que de l'orthographe standard, telle qu'elle apparaît sur le net avec ses erreurs comme ici : tu te prends sans « $\mathrm{s}$ ", « matlatom » pour « matelas Tom ». De même nous avons conservé les néologismes.

8. En majuscules dans le texte.

9. Voir à ce propos Homi K. Bhabha (2007) que cite R. Meran (2008).

http://www.akkun-africa.com/articles-sur-la-bd-africaine/gate-gate/58-gate-gate/313-nd6gategate.html

http://www.akkun-africa.com/articles-sur-la-bd-africaine/gate-gate.html

\section{RÉSUMÉS}

Cette contribution témoigne d'une expérience de collecte et d'analyse d'un genre d'origine oral intitulé gate-gate consistant en une joute sous forme d'échanges rituels d'insultes, genre normalement pratiqué par des jeunes gens dans les milieux urbains de Côte d'Ivoire, mais aussi sur le web par les membres de la diaspora ivoirienne. L'article analyse l'évolution du genre lorsqu'il s'est transporté sur le net; passant de l'oral à l'écrit, mais conservant cependant un certain nombre de ses traits d'oralité. À partir de ce cas particulier est envisagée une réflexion sur la problématique spécifique impliquée par Internet comme nouveau terrain d'enquête possible de genres de la littérature orale, notamment par les conditions d'enquête que ce nouveau media impose. 
This contribution describes the gate-gate, a genre with origins in oral tradition consisting of a ritual exchange of insults. This duel is normally practiced by young men in the cities of Ivory Coast, but it is also exchanged on the web by members of the Ivorian Diaspora. This article analyses the evolution of the genre once it has been transposed onto the Net, passing from oral to written expression but keeping certain oral traits. Based on this particular case, the author reflects on the specific issues raised by Internet as a new field area for collecting oral literature.

\section{INDEX}

Thèmes : anthropologie (Afrique)

Mots-clés : gate-gate, insultes rituelles, Internet, situation d'énonciation

Index géographique : Côte d'Ivoire

Keywords : Context of Communication, Gate-Gate, Internet, Ivory Coast, Ritual Insults, Anthropology 